\title{
LIBERALES, ERUDITAS O SUMISAS: \\ LA REPRESENTACIÓN DE LAS ESPAÑOLAS DURANTE \\ LA DICTADURA DE PRIMO DE RIVERA EN SPANISH PRELUDE DE JENNY BALLOU*
}

\author{
María Losada Friend \\ Universidad de Huelva
}

\section{RESUMEN}

Dentro de la conocida tradición de autores ingleses y norteamericanos que vivieron y describieron la historia de España durante la primera mitad del siglo xx, la obra de Jenny Ballou registra la calma tensa que precede a la Guerra Civil española en los últimos días de la dictadura de Primo de Rivera. Ballou, cuya representación ha sido comparada con George Orwell, recrea en Spanish Prelude (1937) un peculiar travelogue personal, periodístico y literario donde recoge retratos de múltiples personajes decodificando sus actitudes, vivencias y emociones en una época paradójica, donde la rutina diaria se mezcla con la gestación del espíritu revolucionario. En tal contexto, este estudio se centra en la contribución de Ballou a la representación realista de la mujer española en un margen que cubre muchas clases sociales, literarias y políticas.

Palabras clave: libros de viajes, mujeres y la dictadura de Primo de Rivera, americanos en España, Jenny Ballou, Spanish Prelude.

LIBERAL, LEARNED, OR SUBMISSIVE:

THE REPRESENTATION OF SPANISH WOMEN DURING PRIMO DE RIVERA'S

DICTATORSHIP IN SPANISH PRELUDE BY JENNY BALLOU

\section{Abstract}

Within the well-known tradition of British and American authors who lived and described the history of Spain during the first half of the twentieth century, the work of Jenny Ballou records the tense calm before the Spanish Civil War in the last days of the dictatorship of Primo de Rivera. Ballou, who has been compared to George Orwell, recreated in Spanish Prelude (1937) a peculiar travelogue with journalistic and literary traces. Portraits of multiple characters decode attitudes, experiences and emotions in a paradoxical time where the daily routine is mixed with the gestation of the revolutionary spirit. In this context, this study describes Ballou's contribution to the realistic representation of the Spanish woman in a range that encompasses different social, literary and political classes.

Keywords: travel writing, Spanish women and Primo de Rivera's dictatorship, Americans in Spain, Jenny Ballou, Spanish Prelude.

DOI: http://doi.org/10.25145/j.refiull.2019.38.010

Revista de Filología, 38; enero 2019, pp. 155-167; ISSN: e-2530-8548 
«It started in the middle of July. There were no announcements, no newspapers, just a whispering in the street and the sound of a woman weeping» (Lee 2014: 168). Tal es el lacónico comienzo del capítulo "War», con el que el viajero Laurie Lee, un inglés a pie por España en 1936, describía el inicio de la dramática Guerra Civil española. Tras él, muchos otros británicos y americanos registraron en primera línea y con ojos extranjeros el conflicto que cambiaría inevitablemente la historia del país. Sin embargo, si bien son múltiples los trabajos y voces que han analizado la visión foránea en la etapa de la Guerra Civil y sus secuelas ${ }^{1}$, el periodo inmediatamente anterior no ha recibido tanta atención, como afirma Probst Solomon (2007: 190). Resultan por ello significativas las contribuciones de los visitantes que relataron los momentos anteriores al drama, desde los años de la dictadura de Primo de Rivera hasta la proclamación de la Segunda República y el estallido de la guerra. En esta época de calma y tensión -paradójicamente denominada «Edad de Plata» (González Calleja 2005: 294, Tamames 2008: 337)- convergen en España autores internacionales cuyos perfiles mezclan en ocasiones las funciones de reporteros, corresponsales o escritores. Sus experiencias se vierten en testimonios sobre la vida cotidiana y sus estilos demuestran que navegan en una retórica donde saben unir detalles propios del periodismo con la percepción literaria de la realidad e incluso con trazos de memorias autobiográficas. Tal puede ser el caso del británico V.S. Pritchett, cuyo recorrido por España (Marching Spain, 1929) muestra la experiencia e intuición que volcó más tarde en sus contribuciones en The New Statesman o en periódicos americanos como The New Yorker o The New York Times.

Otro caso lo conforma el numeroso grupo de reporteros británicos y norteamericanos que enumera Berga (2018: 16) y que incluye a Hemingway junto con una nutrida representación de mujeres reporteras, o las mujeres americanas periodistas en la Guerra Civil del detallado estudio de Valis (2017). Este tipo de perspectiva crítica arroja luz sobre la presencia femenina en este ámbito y nos permite reflexionar sobre contribuciones valiosas como las de la reportera americana Martha Gellhorn, corresponsal del Collier's Weekly, o de Gamel Woolsey, autora americana de Death's Other Kingdom (1939), cuyos trabajos pueden ser ya hoy reconocidos con independencia de haber sido esposas de Ernest Hemingway o Gerald Brenan, respectivamente.

La escritora y crítica Jenny Ballou (1903-1940), muy unida a la profesión de los corresponsales extranjeros y al mundo periodístico, que conoció en España junto a su marido (Harold Ballou, del American International News Service for the New York Evening Journal), resulta otro eslabón importante en esta época gracias al

* Este trabajo se enmarca en el contexto de la investigación correspondiente al Proyecto I+D La vida emocional de las mujeres: Experiencias del mundo, formas de la sensibilidad. Europa y América (Agencia Estatal de Investigación (AEI) proyecto I+D HAR2015-63804-P).

1 Véanse, entre otros muchos, Constante González Groba et al. (eds.) Travelling Across Cultures (2000), las publicaciones sobre los brigadistas internacionales en España de Rodríguez Celada et al. $(2006,2007,2014)$ o las publicaciones resultantes del proyecto dirigido por Luis Alberto Lázaro Lafuente «El impacto de la Guerra Civil española en la cultura y la literatura de habla inglesa: los textos olvidados» (2014-2016). 
recuento de su estancia en España en Spanish Prelude $(1937)^{2}$. La obra resulta una radiografía de los años previos a la proclamación de la Segunda República. Premiado en su día con el Houghton Mifflin Literary Fellowship, el relato puede considerarse puente entre Hemingway y Orwell y la posiciona en la tradición femenina de otras importantes escritoras que publican esos mismos ańos, como la británica Nancy J. Johnstone (Hotel in Spain, 1937 y Hotel in Flight, 1939), la americana Anna Louise Strong (Spain in Arms y "People of Spain» 1937) o la irlandesa Kate O'Brien (Farewell Spain, 1937). Como ellas, sigue la línea de escritoras extranjeras que reviven el pasado de España en torno a la Guerra Civil, a las que Jackson ha denominado «constructors of the past» (2014: 45) y que convirtieron además la experiencia en parte de su identidad:

... when Spain became a gravitational field attracting a mass of people, women were drawn within its orbit, not only because it was the "cause» of the day, but because for each of them, the conflict was a reflection of their varied concerns. The extension of these concerns to Spanish issues was therefore a somewhat predictable, rather than a predestined, response (45).

En efecto, esta obra no solo permitió a Ballou rememorar su experiencia de cuatro ańos vividos en España - «It is a record of a personality» (Rukeyser 1937: 34)-, sino también potenciar su posición como escritora comprometida, reflexionando desde la distancia sobre los años compartidos con un sector de la sociedad española que parecía esperar a que los acontecimientos se precipitasen. Denunció su estatismo definiéndolo como "that almost incurable form of Spanish Oblomovism» (Ballou 1937: 175)3. Spanish Prelude puede entenderse como el eslabón que confirmó su identidad y actitud comprometida con la causa republicana que potenciaría a su vuelta a Estados Unidos. En efecto, en respuesta al conflicto que aún seguía vivo en Espańa, fue una de las firmantes del conocido documento Writers Take Sides on the Spanish War (Left Review 1937; Calver 2014), donde con otros 400 autores contestaba a la pregunta de Nancy Cunard eligiendo entre "FOR the Government», "AGAINST the Government» o "NEUTRAL» apoyando a la República (Jackson 2014: 138). Su postura, coherente y en la línea de las reflexiones en el libro, se puede leer justo detrás de la respuesta de W.H. Auden y en la misma página que Samuel Beckett, donde la autora afirma:

${ }^{2}$ Los datos más fidedignos encontrados sobre la autora americana son los de Probst Solomon, que informa sobre su nombre de soltera (Iphigenia/Genia/Geni Duphin), su origen ruso y su ingenio (2007: 185). Homenajeó su obra incluyendo el segundo capítulo de Spanish Prelude en su edición de Reading Room/7 en 2007. También resulta informativa la página web de artistas y escritores (http:// lakechapalartists.com), que resume la vida del hijo de Harold y Jenny Ballou, George Adin Ballou, nacido en Madrid en 1927. María DeGuzmán señala antecedentes americanos de los Ballou en Nueva Inglaterra (2005: 243), aunque lógicamente se aplicarían a la familia del marido de la autora y no a ella, nacida en Rusia y residente en EE. UU. desde los tres años.

3 Todas las referencias a Spanish Prelude se han tomado de esta edición. 
I AM FOR THE LEGAL GOVERNMENT and People of Republican Spain and against Franco and Fascism, against the cultural night these names spell. The struggle in Spain has X-rayed the lies upon which our civilisation is built. Those writers who pretend not to see are, at the risk of their personal destruction as artists, betrayers of their own kind (Left Review 1937: 4).

La difusión de Spanish Prelude fue además relevante. El premio que recibió se anunció en España ${ }^{4}$ y la obra fue elegida como anuncio para la portada del Houghton Mifflin Catalogue Spring 1937, donde entre otros se incluía la edición de las Best Short Stories 1937 con obras de Katherine Ann Porter, Faulkner y Hemingway. También diversas reseñas positivas señalaron y avalaron las claves de su éxito: «Its grace, the sharp exciting pictures of Spain» (Rukeyser 1937: 34).

La naturaleza de la obra es, cuando menos, peculiar. Benedict apuntó la dificultad para definir su género: «The book is patently not straight autobiographical narration, neither is it fiction; perhaps it can best be called an abstraction of personal experiences» (1936: 10). Rukeyser la presentó como «simply an impression of Spain» (1937: 33). DeGuzmán la considera un texto moderno fuera del canon con un «significant degree of resistance within the limits of its aesthetics» (2005: 244) y Probst Solomon la define en su totalidad como «one of the most penetrating first-person accounts I had come across» (2007:190). La obra sin duda cabalga entre géneros, entre libro de viajes, memoria autobiográfica y ficción. Una curiosa combinación que incluso alabó la crítica cuando, después de su aparición en Nueva York, se publicó en Londres, donde las reseñas literarias asumieron también la dificultad de su carácter plurigenérico: «It is not precisely a novel. Some could place it on the travel shelf, some among contemporary history, other among essays. Perhaps all would be right» (Sunday Mail 1937: 35)5. Tal colisión de géneros permite contemplar la revisión de sucesos, personas y lugares auténticos bajo el espíritu crítico y reivindicativo de la escritora a través de las experiencias de una narradora alter ego donde el retrato de emociones se construye con éxito (Losada 2017: 338).

Ballou crea así un género propio, enmarcado en el modelo de los libros de viaje con episodios. Contiene dosis de la tradición del roman à clef, puesto que mezcla figuras reales con otras ficticias que guardan estrecha relación con personalidades o tipos del momento ${ }^{6}$. Consigue unir literatura y periodismo siguiendo la tradición

\footnotetext{
4 «La señorita Jenny Ballou, novelista americana, acaba de obtener uno de los premios de mil dólares, creados por el editor Houghton Mifflin, de Boston» (Gaceta del Libro, agosto 1935: 9).

5 Esta reseña iba dirigida a los «British and Australian readers» (1937: 35). Resulta significativo que en otro periódico australiano, la peculiaridad del género de la obra de Ballou se comentara también: «It is a connected series of studies - which might be fiction strongly flavoured with autobiographical data, or might, on the other hand, be well-written fictional reporting of the Spain of de Rivera's dictatorship. Whichever is correct is immaterial» (Sydney Morning Herald 1937: 6).

${ }^{6}$ Benedict definió las fronteras entre la realidad y la ficción en la obra afirmando: «One guesses that the narrator is imaginary, and that some of the characters are compilations and some simple transpositions from life to writing. But all this uncertainty in no way provides a snag» (1937: 10). Rukeyser puntualizó: «students and public figures are alive» (1937: 33).
} 
de Hemingway y Orwell. Como en Fiesta (1926), adopta la máscara de una voz narradora norteamericana que va relatando su vida y encuentros tomando parte en la acción. Así, transcurre la obra describiendo la Sierra de Guadarrama, donde en el pueblo de Anselmo se anuncia a gritos la prensa amarilla con el caso del crimen de Segovia por cinco céntimos; o se visualiza el Madrid de los tumultos estudiantiles, de los cafés o de las pensiones de Gran Vía. Se describen las visitas a la cárcel, al hospital de El Escorial o el tren que lleva a la Exposición Universal de Barcelona y a la Iberoamericana de Sevilla. Ballou consigue simultáneamente recoger los rasgos de otra generación perdida, la generación española de la burguesía intelectual y sus sirvientes amenazada por una calma tensa que magistralmente desgrana. También, en la línea de Orwell en su Homage to Catalonia (1938), Ballou representa en clave activista, profética y en retrospectiva el tiempo previo, presente y posterior a la contienda; con distancia sopesa las ilusiones e inocencias perdidas de una época que inevitablemente estaba dando paso al drama ${ }^{7}$.

Se revisan los años en los que el destino de España se gestionaba entre Primo de Rivera y el rey Alfonso XIII, desde septiembre de 1923 a enero de 1930. Se incluyen en retrospectiva sucesos que ocurrirían antes de la proclamación de la República, el 14 de abril de 1931, englobando también la denominada «dictadura blanda» del general Berenguer y periodo del almirante Aznar. Es el periodo del declive de la dictadura y la inminente salida del rey del país. Por ello, Ballou selecciona escenas que describen un monarca humillado, las revueltas estudiantiles donde la Guardia Civil se convierte en el centro de la burla o hechos que ponen de manifiesto la crisis en ciernes. Describe el paso lento de la vida cotidiana y su sorpresa ante la calma y pasividad de los intelectuales y la acomodada burguesía que se preparaba para amoldarse a la República. La narración potencia el retrato de la intrahistoria en personajes que van de lo más real a lo más alegórico y que permite observar, sobre todo en la galería de personajes femeninos, comportamientos, actitudes y obligaciones que rodeaban a la mujer española de la época. Ballou recoge en parte el hecho de lo que Mangini (1995) prueba en su estudio, el lento pero imparable proceso de visualización de la mujer en los años anteriores a la Guerra Civil ${ }^{8}$. La atención a los personajes femeninos es deliberada y permite un estudio de los mismos en tres niveles: nivel real, imaginado y alegórico.

La narración contextualiza los sucesos que cargaban el ambiente con signos de inestabilidad. Los símbolos del poder político empezaban a derrumbarse y la narración lo acusa. Se recoge la quema de un retrato de Alfonso XIII y la fotografía de Primo de Rivera hecha trizas en la Puerta del Sol (74), se menciona el levantamiento

Probst la compara con Orwell por haber sido «an extraordinary acute witness for the times she lived through» (2007: 191).

${ }^{8}$ Mangini prueba que se había heredado de la Primera República (1873-1874) el espíritu de la liberalización de la educación con el Instituto Libre de Enseñanza y una ideología que veía posibilidades en el potencial de la mujer y sus actividades. Apunta el ascenso de la mujer a la vida pública pese al numeroso grupo de mujeres sin educación, el desarrollo textil de los años 30 o las fundaciones culturales antes de la Guerra. 
de oficiales, "one of these uprisings that were like improvised rehearsals leading to the general dress rehearsal of 1931" (74). Hay detalladas revueltas de estudiantes: «The student fracas» con la carga de la Guardia Civil a estudiantes que encendían cerillas entonando «We are looking for our constitutional rights!» (73), o la manifestación en contra del ministro de Educación de Primo de Rivera donde marchaban a cuatro patas con el cartel "YO SOY UN BURRO» (41). En este contexto entran en el retrato personajes femeninos que alcanzaron prominencia en la vida política y cultural del momento. Por ello no es extraña la aparición de Vitoria Kent visitando a Álvaro de Albornoz en la cárcel, donde más tarde entra Valle Inclán. El libro no deja pasar la presencia de esta abogada y directora general del sistema de prisiones en 1931 que destacó por su labor reformista y por la creación del Cuerpo Femenino de Prisiones. También aparece Isabel García Lorca, hermana pequeña del poeta ${ }^{9}$ y clave fundamental para la Residencia de Señoritas y las Juventudes Universitarias. Otras referencias como a la escritora Concha Espina confirman la voluntad de Ballou de dejar constancia de la voz intelectual femenina, la denominada «República de las Mujeres» (Cruz 2014: 203).

Hay dos espacios importantes en la obra que reúnen al colectivo del ámbito burgués. Son los que dan título al segundo y al tercer capítulo, "Café Revolutionaires» $\mathrm{y}$ «The Women's Club», los más extensos en el libro y donde la crítica se vuelve exacerbada. Son los lugares donde la narradora describe dos protagonistas (Teresa Baranada y Carmen), a las que dedica otros sendos capítulos. Ambas son el retrato de pseudoliberales que participan de la vida social y cultural y que supuestamente están comprometidas con el republicanismo sin perder sus privilegios y vida de burguesas. Son dos mujeres complejas, de acciones contradictorias y más inclinadas a la pose que al compromiso real.

«Café Revolutionaires» refleja el mundo de la vanguardia de Madrid, sus asistentes (identificados indistintamente con los términos «advancedguardists» y «vanguardists») que se retratan inmovilizados en una atmósfera de "mortal boredom» (69), donde aún la presencia femenina se consideraba excepción, tal y como refiere la narradora:

I was rewarded with an astounding sight. In the corner table where, since the closing of the Ateneo, Ramón del Valle Inclán delivered his midnight monologues to a circle of hibernating intellects, sat Teresa Baranada with several vanguardists. It was the only time I had ever seen a woman among them. For although Teresa collaborated in their papers, they never acknowledged her or asked her to join them in their manly round-table discussions (69).

Este café es la contrapartida de los muchos cafés que desarrollaron, como alternativa al Ateneo de Madrid, un "great republican debating club» (Casanova

9 Probst menciona la conexión entre Ballou y Lorca con la presencia de la autora en el grupo de intelectuales (con Herschel Bricknell, Olin Downes, Federico de Onís y Mildred Adams) que se reunió en el barco en la ruta de Lorca desde Puerto Rico a Espańa por Nueva York (2007: 191). 
2015: 15) que fue cerrado en 1924 y abierto de nuevo en 1926 con una Junta Directiva afín a la dictadura (González Calleja 2005: 295). La narradora es testigo de conversaciones triviales y de poca profundidad ( $\mathrm{O}$ ne of them was boasting that there was a certain shade of purple that positively gave him toothache», 69). Impera un espíritu de indolencia que banaliza las preocupaciones y que solo ofrece ocurrentes respuestas ante las novedades, como la aparición de un periodista americano. Es un colectivo que se describe duramente como «an incestuous little bourgeosie of their own» (66) y que revela una élite apartada de la realidad. En este ambiente, Teresa es el tipo de mujer burguesa fría y con pose liberal impregnada del «cruel langor» (69) del grupo vanguardista, pero suspicaz ante injerencias internacionales. Carmen, a su vez, presenta una educación religiosa que será uno de los rasgos que más explotará la narradora. Sus manos ("very white and flat-tipped», 70) unidas como en oración acostumbran al lector a visualizarla en el aura religiosa que la define: «Carmen was looking down at her white knitted gloves which she had interlocked near her beerglass, in a gesture of prayer. She did not lift her eyes» (71). Se las presenta como mujeres seguidoras del utópico mensaje de Ortega y Gasset ("their patriot saint») ${ }^{10}$. Son discípulas del intelectual, de la figura creadora de un discurso preocupado por España pero alejado de la realidad. Al autor se le admira su fuerza intelectual, pero se le ataca por promover una revolución edulcorada, «a polite revolution», «a corteous revolution" (81). La narradora no encuentra en este colectivo trazos de la inquietante situación en la que viven: «This was the first time I had heard the word 'future' in Madrid, although I had been there three years. As to 'revolution' the nearer it approached in actuality, the less it was heard about in the cafés» (71).

Las mujeres representadas forman parte de este grupo moderno sin preparación para el drama que se avecina. Se pone de manifiesto que están al tanto de novedades literarias como las de Gómez de la Serna, descrito como «genial semigod of the vanguardists» (66), que no serán útiles después de la República, sino meros «experimenters of the New Spain» (68). Descrito como «experimental souls», este grupo burgués es en realidad el foco del ataque de Ballou, que promueve que la cortesía o la inocencia no son armas para liderar la revolución necesaria. Por ello, el retrato de las indulgencias frívolas de las dos mujeres descritas se detalla con cuidado y pone de manifiesto el desarrollo de los productos cosméticos y de lujo que se iban perfeccionando en la época, como las medias de seda, el lápiz de labios, los coches,

${ }^{10}$ Este retrato se une con comentarios sobre documentos reales que revisan el discurso de Ortega en el Pen Club publicado en Revista de Occidente (diciembre de 1935). La narradora denuncia su único interés por «his revolutionary philological problems» (82) y cita serios enfrentamientos futuros que ocurrirían más tarde, como los granjeros golpeados en prisiones, trabajadores en situación trágica en Asturias o la matanza de Casas Viejas de 1933. Sin embargo, el final del capítulo equilibra esta negativa imagen («Ortega himself, in the final test, proved that his actions were worth more than his words», 83), lo que muestra el conocimiento que tenía Ballou de la decisión de Ortega en febrero de 1931, del rechazo de la élite intelectual al rey y la fundación con Marañón y Pérez de Ayala de la Agrupación al Servicio de la República (Hall 2005: 376). Para la evolución del perfil del intelectual ver también Gracia (2014). 
etc. (González Calleja 2005: 269). La narradora no perdona la tibia posición de este grupo intelectual y ataca la poca consistencia de los valores de la vanguardia ante los cambios: "They carried their vanguard burlesque into the new Republic, of which they were destined to become the playboys» (67). Con todo ello, se entiende la intencionada ironía del calificativo "Revolutionaires» para el título del capítulo.

Otro espacio real que se recrea en la obra es The Women's Club. Es claramente la contrapartida del auténtico Club Lyceum Femenino creado y presidido en 1926 por María de Maeztu como el primer club de mujeres en Madrid (con Victoria Kent e Isabel Oyarzábal como vicepresidentas). Contó en 1930 con casi 500 miembros y abrió una institución similar en Barcelona. Las escenas en el libro contextualizan la respuesta de Maeztu a la injerencia de grupos conservadores sobre las actividades del Club:

The Club had opened the year before with the announcement that it was formed by the wives of the famous men of Spain. But the President, unmarried, was famous herself, and very ambitious. Her hair disarranged, her hat fallen carelessly on one side, and her face congested, she was threatening to resign. There was some kind of discussion, if it could be that, about an attack made upon the Club by some priests who had accused the Club of being an immoral institution with pagan ideas about the freedom of women imported from Soviet Russia that lured home-makers from their hearths, separated husbands and wives, and made mothers strangers to their own offsprings (107).

Se revela así la oposición conservadora a la actividad dinámica que tenía el Club, pero la incisiva narradora va más allá, presentando una congregación que no parece interesada («as I looked about me I did not see anybody who cared one way or the other what was decided») o que se deja llevar por las decisiones que se toman por la dirección ("The members bleated a unanimous 'Aye' and rushed to the tea-room», 108).

La actividad frenética que fue la base del éxito del Club Lyceum en su larga andadura de diez años se basaba en parte en la asistencia a seminarios de Derecho o visitas de importantes conferenciantes. De ahí que la narradora registre una pomposa entrada de Alberti, descrito con aguda sorna:

As soon as Alberti appeared, young, dashing, and enhanced by his disdain for the women to whom he was to read his poems, the members went back to the drawing-room that was now filled with guests. The poet started with a preliminary talk against all the famous husbands of the members (108).

Esta asamblea retrata -aunque de manera paradójica- el deseo de cultura, de espíritu de defensa de la igualdad femenina y de fusión con otros liceos que por toda Europa se unían para el desarrollo de la lucha por el sufragismo. Sin embargo, la narradora no obvia el hecho de que parte de esta burguesía ilustrada femenina no parecía percibir la amenaza de un futuro dramático y define personajes con un concepto de liberalismo de pose que no comparte. Recorren los capítulos personajes como Julia («an unfunctioning writer»), quien, perteneciente por nacimiento a la nobleza 
y casada con un crítico de arte pusilánime, es un espíritu despreocupado de la realidad o del paso del tiempo: «She held the passing years casually, and her face, with its halo of light hair, was quite free from the desperate attempt at youthfulness that characterized the other women in the Club» (109).

De todo este cuadro, la narradora solo vuelve a América recordando la buena impresión de un personaje interesante, Esperanza, prima de Carmen y muy diferente al resto precisamente por no pertenecer al mismo nivel económico: «I was at that time aware of a reality in Esperanza that I missed in my other friends» (173). Es el único personaje que aprende a mirar con los ojos que impone la narradora. Esperanza reconoce ser «not very determined, not very vital» (174) como el resto de sus compatriotas y la narradora recibe esta confesión como una revelación que le permite reflexionar sobre las posibilidades de salvación de España. En los últimos capítulos sopesa el valor de la República y se alegra al descubrir a Esperanza en una fotografía del periódico, en un grupo de chicas preparadas para luchar en la guerra junto a Dolores Ibárruri, «La Pasionaria», a la que define por su «powerful face of her countrywoman» (175).

Otro grupo de mujeres representadas es el que abarca todo un universo de sumisión que parece envolver a los personajes femeninos de la obra. La narradora recorre retratos y acciones enfrentándose al pasado de muchas mujeres y parece comprender las líneas de poder y desigualdad que son parte de la vida cotidiana femenina. Al igual que se retratan los modelos de modernidad en comunicaciones, ocio, deportes, diseños interiores y pisos encerados que lentamente se introducían en España con el lento acceso de la mujer al trabajo público (González Calleja 2005: 273), también se presentan retratos femeninos y tipos que muestran aspectos múltiples de una generación que vivía entre el acatamiento a la tradición y los deseos de independencia.

Frente a las intelectuales o pseudoliberales retratadas, la narradora contrasta el grupo de mujeres de la familia del doctor Monteagudo, que visita en la sierra de Guadarrama, en Madrid y en El Escorial. Son parte de una sociedad rancia y conservadora. Doña Rosa, madre y viuda, ha aprendido a guardar silencio ante la posición de su hijo como hombre de la familia. Es él quien denomina a su propia hermana «la beata» ("lightly teasing her about her endless devotions», 90), otra viuda que vive envuelta en olores de moho, aguarrás e incienso y que anuncia a gritos la revolución cuando oye la explosión de una rueda de coche en la Castellana. Carlota, la prima del doctor, comprometida, espera lánguidamente tras tres ańos de enfermedad para casarse. Su soledad, su sombrío jardín, su voz monótona y sus horas frente al espejo dejan en el libro otra pincelada de inmovilismo y sumisión a la tradición.

Frente a esta clase acomodada e inmóvil contrastan los excelentes retratos del universo de las trabajadoras, con las que la narradora observa también otras variedades de sumisión. Margarita Vaquero regenta activamente la pensión soleada donde la narradora vive sus primeros momentos en Madrid. Su modernidad externa se revela en su corte de pelo y sus complementos a la moda («her dark bobbed hair [...] and her modern tortoise-rimeed glasses», 47). Sin embargo, su sumisión ante las exigencias del canon de belleza de la delgadez y la cosmética del momento (González Calleja 2005: 269) causan su perdición («Well, all that just to be slender!», 50). 
Abandonada por su marido, se inclina sumisa y fervorosa a su amante, quien, despótico, le recrimina su infertilidad (49). En Saturnina, la criada llegada de la sierra, encontramos la sumisión de la incultura y de la inexperiencia ante todo lo moderno:

She was afraid, afraid to go down on the elevator, afraid to be taken for a country girl on the streets, afraid to pass the doorman on her return to the market, who with his uniform, was as abstract, as unbearably mythological as all the rest. (100)

Celia, la costurera, es una chica preparada e inteligente («she came to me directly from the recluse poet Juan Ramón Jiménez», 101), pero es consciente de la rigidez de las clases sociales. Sueña con la parafernalia de los ricos y aprende a hacer de la ropa su signo de identidad, sumisa ante su estatus inamovible entre la burguesía y las clases menos pudientes: «Although she did not go so far as to wear a hat, when she went on the street she wore a vague little veil to mark her difference from the forms of service which she considered beneath her station» (101).

Estas son unas meras pinceladas de la rica galería de mujeres con las que la narradora retrata la necesidad de educación y de independencia económica, carencias de esta sociedad a la que acechaba un espíritu de revolución que, según las claves de Ballou, no se había asimilado. La narradora observa esta actitud de acatamiento y mansedumbre en otras muchas de las mujeres que retrata: en prostitutas que se dejan arrastrar a los cafés intelectuales ("that unmistakeable humility and wifeliness of a Spanish prostitute», 69), en la joven empujada por su propia madre a convertirse en amante del «Príncipe X» (108), en mujeres con maridos amenazadores o con la presencia de algún «violent woman-hater» (54).

Rebasando el nivel de lo real o lo representado, Ballou introduce, por último, el nivel alegórico, que debe entenderse como aquel donde eleva el registro de viajera a una dimensión transcendente y espiritual. Ocurre en las reflexiones finales con el caso de uno de los personajes que adquiere trazos simbólicos. Es la tía Mauricia, que llora desconsoladamente al principio de la obra cuando pierde su vaca. A ella se hace alusión al final, cuando la narradora deja abierto un rayo a la esperanza para España, entonando un deseo casi desesperado por el cambio, donde no solo la tía Mauricia podría encontrar consuelo, sino la sociedad española la renovación que necesita: «All will have running water in the new age of Spain, and la tía Mauricia will have her cow, and there will be no masters and no servants and no city and no country» (305).

Con este deseo de cambio que cierra Spanish Prelude y como conclusión, resulta oportuno evocar dos importantes aspectos que Roberson define como clave teórica en la escritura de viajes de mujeres americanas, diferenciando entre «ideological mobility» y "geographic movement» (2009: 225). Tal distinción nos ayuda a entender que el movimiento geográfico del viaje de Ballou a España impulsó y reactivó su movilidad ideológica, capturando experiencias, definiendo su abierto rechazo al fascismo y dejando clara muestra de su activismo como escritora y crítica en favor de la causa republicana. El libro de Ballou muestra que conoció y asimiló bien diferentes ambientes periodísticos, culturales e intelectuales, incluso estuvo registrada en la Residencia de Estudiantes, como recoge Sáenz de la Calzada (1986: 
180). Supo además seguir los avatares de la vida española haciéndola visible al lector americano a través de sus interesantes ideas y reseñas de libros sobre poemas de García Lorca, sobre novela, sobre la música en obras de Falla o de jóvenes promesas como Ernesto Halffter, o sobre la pintura de Gutiérrez Solana. En todos ellos, y sobre todo en Spanish Prelude, da muestra de la atracción por un país que observa con atención detallando su inevitable paso hacia la modernidad en un momento de cambio inminente y preocupante. Por ello, el valor de la obra de Ballou reside no solo en el registro casi periodístico de los rasgos reales de la Espańa de finales de los ańos 20 y de los 30 , sino en la recreación literaria de un viaje al exterior convertido en viaje interior que reavivó su compromiso con las culturas española y norteamericana.

Recibido: junio de 2018; aCePtado: octubre de 2018. 


\section{BIBLIOGRAFÍA}

Ballou, Jenny (1937): Spanish Prelude, Boston: Houghton Mifflin.

Benedict, Libby (1937): «Overtones of Spain», The Saturday Review, 15: 10.

Berga, Miquel (2018): «Prólogo» a Virginia Cowles, Complicarse la vida, Barcelona: Tusquets, 13-19.

Book Publishing Institute (2016): «Houghton Mifflin Catalogue 1937». URL: http://bookpublishinginstitute.org/2013/08/05/houghton-mifflin-spring-1937-trade-catalog/; 05/06/2016.

Calver, Katherine Elizabeth (2014): Authors Take Sides on the Spanish War: A Dossier. Ph.D. Dissertation, Boston University.

Casanova, Julián (2005): Anarchism, the Republic and Civil War in Spain: 1931-1939, London and New York: Routledge.

Cruz, Rafael (2014): Una revolución elegante. España 1931, Madrid: Alianza Editorial.

DeGuzmán, María (2005): Spain's Long Shadow: The Black Legend, Off-Whiteness, and Anglo-American Empire, Minneapolis: University of Minnesota Press.

González Calleja, Eduardo (2005): La España de Primo de Rivera, Madrid: Alianza Editorial.

González Groba, Constante et al. (eds.) (2000): Travelling Across Cultures/Viaxes Interculturais: The Twentieth-Century American Experience, Santiago: Universidade de Santiago de Compostela.

Gracia, Jordi (2014): José Ortega y Gasset, Madrid: Colección Españoles Eminentes.

Hall, Morgan (2014): Alfonso XIII y el ocaso de la monarquía liberal. 1902-1923, Madrid: Alianza Editorial.

Hemingway, Ernest (2004) [1926]: Fiesta or The Sun also Rises, London: Arrow Books.

JaCkson, Angela (2014): British Women and the Spanish Civil War, London and New York: Taylor and Francis.

Lee, Laurie (2014) [1969]: As I Walked Out One Midsummer Morning, London: Penguin Classics.

Left Review (1937): Authors Take Sides on the Spanish War, London: Purnell \& Sons.

Losada Friend, María (2017): «A Social, Political and Emotional Travelogue on Pre-Revolutionary Spain: Jenny Ballou's Spanish Prelude» en Julio Cañero (ed.), North America and Spain: Transversal Perspectives, New York: Escribana Books, 329-340.

Mangini González, Shirley (1995): Memories of Resistance: Women's Voices from the Spanish Civil War, New Haven and London: Yale University Press.

Orwell, George (2000) [1938]: Homage to Catalonia, London: Penguin Classics.

Pritchett, V.S. (1988) [1928]: Marching Spain, London: Hogarth Press.

Probst Solomon, Barbara (ed.) (2007): The Reading Room/7. Writing of the Moment, New York: Great Marsh Press, 189-191.

Roberson, Susan L. (2009): «American Women and Travel Writing», en Alfred Bendixen y Judith Hamera (eds.), The Cambridge Companion to American Travel Writing, Cambridge: Cambridge University Press, 214-227.

Rodríguez Celada, Antonio et al. (eds.) (2006): Los brigadistas de habla inglesa y la Guerra Civil española, Salamanca: Ediciones Almar. 
Rodríguez Celada, Antonio et al. (eds.) (2007): Las Brigadas Internacionales: 70 años de memoria histórica, Salamanca: Amarú Ediciones.

Rodríguez Celada, Antonio et al. (eds.) (2014): La prensa británica y la Guerra Civil española, Salamanca: Amarú Ediciones.

Rukeyser, Muriel (1937): «Such as Proust?», New Masses, 4 May: 33-34.

Sáenz de la Calzada, Margarita (1986): La Residencia de Estudiantes 1910-1936, Madrid: CSIC.

Strong, Anna Louise (1937): «People of Spain», New Masses, 26 January: 4-6.

Strong, Anna Louise (1937): Spain in Arms, New York: Holt.

SUNDAY MAIL (1937): «Prelude to Civil War in Spain. Glittering Picture of Strange Land of Beauty and Barbarity", 25 July: 35.

SYDNEY MORNING HERALD (1937): «Pre-Revolutionaly Spain», 20 August: 6.

Tamames, Ramón (2008): Ni Mussolini ni Franco: la dictadura de Primo de Rivera y su tiempo, Barcelona: Planeta.

VAlis, Nöel (2017): “"From the Face of My Memory”: How American Women Journalists Covered the Spanish Civil War», Society 54 (6): 549-559. 
